\title{
Preliminary study to determine the phenolic maturity stage of grape seeds by computer vision
}

\author{
Francisco J. Rodríguez-Pulido ${ }^{a}$, Raúl Ferrer-Gallego ${ }^{\mathrm{b}}$, M. Lourdes González-Miret ${ }^{\mathrm{a}}$, \\ Julián Carlos Rivas-Gonzalo ${ }^{\mathrm{b}}$, María Teresa Escribano-Bailón ${ }^{\mathrm{b}}$, Francisco J. Heredia ${ }^{\mathrm{a}, *}$ \\ a Food Colour \& Quality Lab., Dept. Nutrition E Food Science, Facultad de Farmacia, Universidad de Sevilla, 41012 Sevilla, Spain

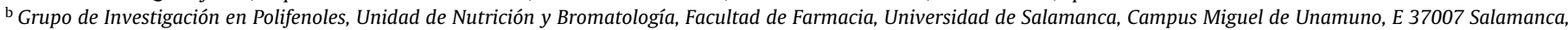 \\ Spain
}

\section{A R T I C L E I N F O}

\section{Article history:}

Received 1 September 2011

Received in revised form

29 December 2011

Accepted 4 January 2012

Available online 10 January 2012

\section{Keywords:}

Image analysis

Colour

Vitis vinifera

Grape seeds

Phenolics

\begin{abstract}
A B S T R A C T
The applications of computer vision technology for acquiring and analysing images have been extended to the quality evaluation in food industry. This technique involves great advantages for the objective, rapid, non-contact and automated quality inspection and control. The aim of this work was to evaluate the potential of the computer vision to determine the phenolic maturity stage of grape seeds. Up to 21 phenolic compounds were determined by HPLC-DAD-MS in order to obtain reference values to develop the model. The CIELAB parameters, area, aspect, roundness, length, width and heterogeneity of seeds were analysed using a DigiEye ${ }^{\circledR}$ system. The technique reported in this work can be a good and rapid tool for taking decisions at harvest time. Notwithstanding, a comprehensive study should be made in order to develop more robust models.
\end{abstract}

(c) 2012 Elsevier B.V. All rights reserved.

\section{Introduction}

The phenolic composition of grape seeds plays an important role on the red wine quality. In this sense, the contribution of seed tannins to several sensorial attributes of wines, such as body, structure, bitterness, astringency and colour are well known [1,2]. Also, the presence of phenolic compounds in wine has been the subject of great interest due to their potentially beneficial effects on human health [3].

Many physical and biochemical changes take place during grape seeds maturity which affect their characteristics like astringency, tannic intensity, colour and morphology [4,5]. Quantity, structure and degree of polymerization of seed tannins are modified during ripening development $[6,7]$. Monitoring these changes could be an important tool for deciding the optimal moment for harvesting, but this would require to have rapid analytical methods involving minimal or no sample preparation.

Colour and appearance are closely related to the chemical composition and sensory properties of food. Usually, colour is determined by tristimulus colorimetry and expressed in terms of $L^{*} a^{*} b^{*}$ variables, corresponding to the uniform colour space CIELAB.

\footnotetext{
* Corresponding author. Tel.: +34 954556495; fax: +34 954557017

E-mail address: heredia@us.es (F.J. Heredia).
}

The CIELAB colour space (1976 $L^{*} a^{*} b^{*}$ colour space) is an international standard for colour measurements, recommended by the Commission International d'Eclairage (CIE) in 1976. Within CIELAB, a psychometric index of lightness $\left(L^{*}\right)$ and two colour coordinates ( $a^{*}$ and $b^{*}$ ) are defined. $L^{*}$ is a qualitative attribute of relative luminosity, which is the property according to which each colour can be considered as equivalent to a member of the grey scale, ranging between black $\left(L^{*}=0\right)$ and white $\left(L^{*}=100\right)$. Coordinate $a^{*}$ takes positive values for reddish colours and negative values for greenish ones, whereas $b^{*}$ takes positive values for yellowish colours and negative values for bluish ones [8]. From these Cartesian coordinates, two angular parameters can be defined: chroma $\left(C^{*}{ }_{\mathrm{ab}}\right)$ and hue $\left(h_{\mathrm{ab}}\right)$ [9]. Hue angle $\left(h_{\mathrm{ab}}\right)$ is the qualitative attribute of colour (blue, purple, green...), and chroma $\left(C^{*} \mathrm{ab}\right)$ is considered the quantitative attribute of colourfulness, allowing assessing the degree of difference of any given hue relative to a grey colour with the same lightness.

The CIELAB colour space is considered as uniform by the CIE, i.e., the Euclidean distance between two different colour points corresponds approximately to the colour difference perceived by the human eye.

For colour measurement, different kinds of instruments such as colorimeters, spectrophotometers and spectroradiometers can be used. However, in the case of non-homogeneous colour, these instruments require homogenizing the sample to achieve uniform 
colour. This fact makes complicated and tedious measuring colour of heterogeneous stuff or small objects, such as grape seeds.

Computer vision provides fast, accurate, direct and nondestructive analysis that this case needs. Moreover, not only colour but also other characteristics such as shape, texture and homogeneity can be determined $[10,11]$.

A computer vision system includes an illumination system, a charge-coupled device (CCD), a frame grabber which converts the analogue image from the camera into a digitized one, and a computer with the suitable software for image processing and interpretation of results [12]. The digital camera receives images onto a CCD that registers them in gradations of three basic colours: red, green and blue (RGB colour space). This colour space is widely used in electronics devices but it depends on the device where it is visualized. So, we cannot use this space for absolute measures; it is necessary to transform the data from RGB to CIELAB colour spaces. This transformation requires calibration and depends on the illumination when images are taken.

In the image analysis perform, it is indispensable to recognize objects into the image before analyse them. For this purpose, the representation of the image is changed into something more meaningful and easier to analyse [13]. This process is known as segmentation and refers to the partition of a digital image into multiple sets of pixels. Once this recognition is done, the morphology of the objects are analysed and colour coordinates are calculated from the pixels that they are composed of. Frequently, HSI (hue, saturation and intensity) space is used for the segmentation process [14]. HSI colour space is easier to understand than RGB due to its likeness with the $L^{*}, C^{*}$ ab and $h_{\mathrm{ab}}$ stimuli, which are more related to the visual appreciation of colour.

The aim of this study was to find relationships between chemical (phenolic composition) and appearance (colour and morphological) data of grape seeds which can yield to the assessment of the phenolic maturation stage based on parameters obtained by image analysis.

\section{Materials and methods}

\subsection{Samples}

Vitis vinifera L. cv. Graciano red grape berries grown on Rioja Designation of Origin (Spain) were collected at two vineyards in 2010, at six different stages during the berry maturation (from veraison until over-ripeness). Sampling was carried out as follows: 150 berries were collected from both sides of vines in a row within the vineyard. Edge rows and the first two vines in a row were avoided. In order to have representative samples, berries were collected from the top, middle and bottom of the clusters from different vines along the vineyard. The samples (whole grapes) were immediately frozen and stored at $-20^{\circ} \mathrm{C}$ until the analyses were performed.

\subsection{Phenolic extraction and analysis}

Phenolic extraction was carried out as described in GarcíaMarino et al. [15]. For extraction, grape seeds were separated manually and freeze-dryed. After this, samples were ground to obtain homogeneous powder. $2 \mathrm{~g}$ of homogeneous powder was extracted with twenty millilitres of methanol:water (75:25). The extraction process was repeated twice more. The methanolic extracts were combined and concentrated at low pressure until aqueous extract was obtained. The extracts were filtered through a $0.45 \mu \mathrm{m}$ filter and injected directly into the chromatographic system. All analyses were performed in triplicate.

Liquid chromatography-diode array detection-mass spectrometry (LC-DAD-MS) analysis was performed using an Agilent
Technologies 1200 series liquid chromatograph (Palo Alto, CA, USA). A Spherisorb ${ }^{\circledR}$ S3 ODS- 2 reverse phase $3 \mu \mathrm{m}$ particle size C18 column $150 \mathrm{~mm} \times 4.6 \mathrm{~mm}$ i.d. (Waters, Ireland) thermostatted at $25^{\circ} \mathrm{C}$ was used. The solvents used were (A) $2.5 \%$ acetic acid, (B) $2.5 \%$ acetic acid/acetonitrile (90:10, v:v), and (C) HPLC-grade acetonitrile, establishing the following gradient: from 0 to $100 \% \mathrm{~B}$ for $5 \mathrm{~min}$, from 0 to $15 \% \mathrm{C}$ for $25 \mathrm{~min}$, from 15 to $50 \% \mathrm{C}$ for $5 \mathrm{~min}$, and isocratic $50 \% \mathrm{C}$ for $5 \mathrm{~min}$, at a flow rate of $0.5 \mathrm{~mL} \mathrm{~min}^{-1}$. Detection was carried out at $280 \mathrm{~nm}$ as preferred wavelength. The mass spectrometer (MS) was connected to HPLC system via the DAD cell outlet. MS detection was performed with API 3200 Qtrap (Applied Biosystems, Darmstadt, Germany) equipped with an electrospray ionization (ESI) source and a triple quadrupole-ion trap mass analyzer, controlled by the Analyst 5.1 software.

Quantification was performed by HPLC-DAD using calibration curves of $(+)$-catechin, purchased from Sigma-Aldrich (St. Louis, USA), and of procyanidin standards previously obtained in our laboratory [16,17].

\subsection{Image acquisition}

The DigiEye ${ }^{\circledR}$ imaging system based upon the calibrated digital camera was used [18]. This device consists on a closed illumination box, specially designed by VeriVide Ltd. (Leicester, UK), and a digital camera (10.2-megapixel Nikon ${ }^{\circledR}$ D80 with Nikkor ${ }^{\circledR} 35 \mathrm{~mm}$ f/2D objective) connected to a computer (Pentium IV $3.00 \mathrm{GHz}$ processor) via USB. The cabinet is equipped with two CIE D65 standard illuminant emulators which allow illuminating the samples consistently under stable lighting conditions. For obtaining morphological and appearance parameters, as well as the CIELAB coordinates from RGB colour space data, the software DigiFood ${ }^{\circledR}$ [19] was used.

\section{Results and discussion}

\subsection{Chemical analysis}

Based on the chromatographic and spectral features, twentyone phenolic compounds were identified. These were quantified and grouped into hydroxybenzoic acids, flavanol monomers (including catechin and epicatechin), dimers of procyanidins, trimers of procyanidins and galloylated flavanols (epicatechin 3O-gallate and galloylated procyanidins). Fig. 1 shows the evolution during ripening of the studied phenolic compounds. These compounds correspond to the main phenolic compounds present in the chromatograms of the seed extracts.

Total phenolic content, defined as the sum of the content of the phenolic compounds identified, decrease during ripening, in agreement with other works [4,6,20-22]. Monomers and galloylated flavanols undergo the sharpest drop. Near maturity, the content of galloylated compounds is close to that found by other authors for this variety ( $24 \%$ of total) [4,23]. The pronounced decrease of the flavanol monomers provokes that the ratio of the concentration of monomers to oligomers (dimers and trimers) tends to diminish during ripening. Thus, values close to 1 can be calculated at veraison while ca. 0.6 is obtained at harvest. This is consistent with possible polymerization reactions occurring during maturation.

Hydroxybenzoic acids are present in seeds in low amounts (from 0.4 to $0.5 \mathrm{mg} \mathrm{g}^{-1}$ ) and their concentration do not suffer as noticeable changes during ripening as flavanols.

\subsection{Image analysis}

Images of the seeds corresponding to 100 berries per sample were acquired by using the DigiEye system. For seed recognition 

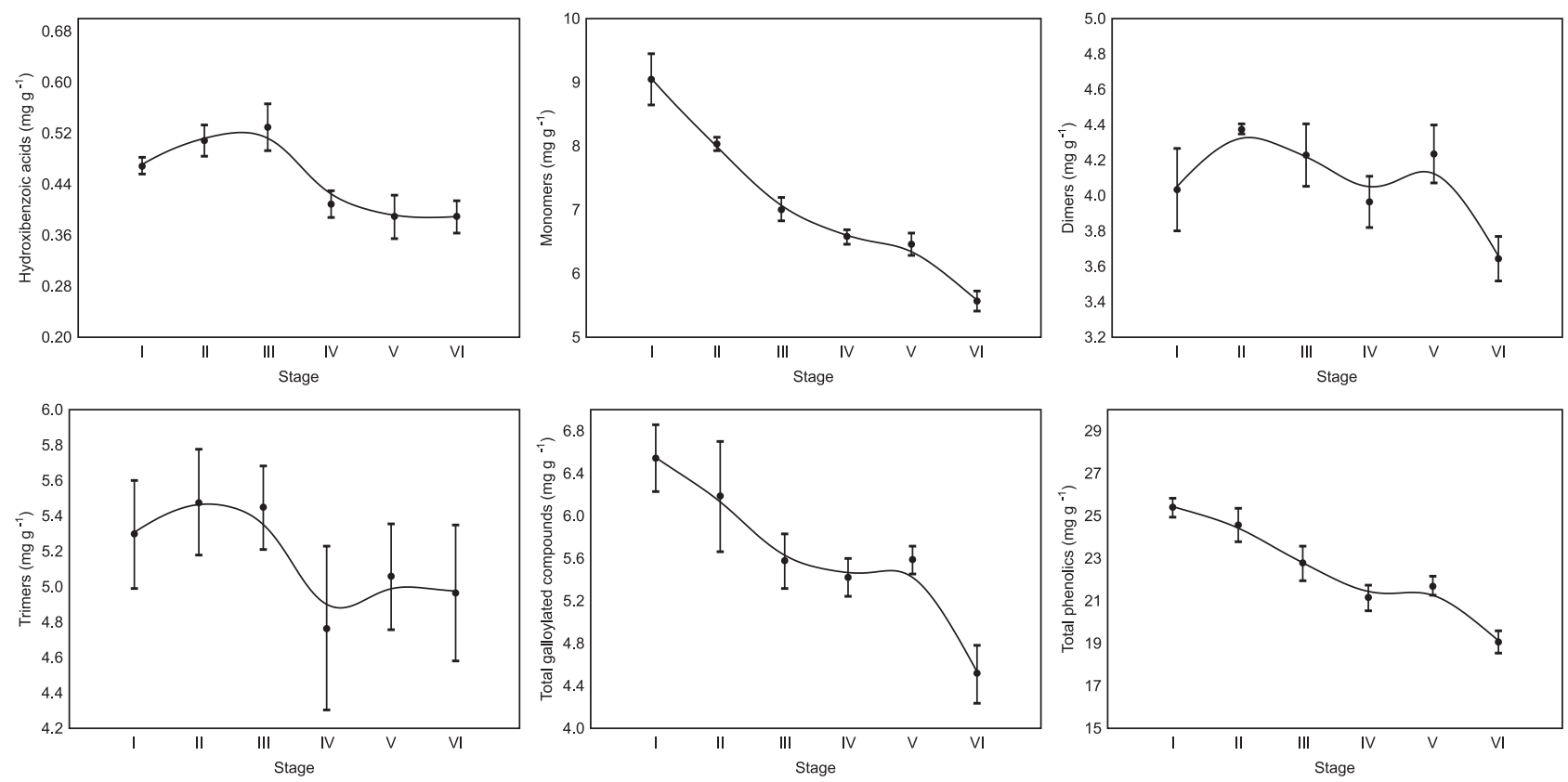

Fig. 1. Contents of the different polyphenols divided in groups at each stage of maturation.

within the images, the following selection criteria were established: (a) regions having values of saturation (S) lower than 180 units, and intensity (I) lower than 140 units, (b) objects having area values between 6 and $35 \mathrm{~mm}^{2}$, and length values less than $15 \mathrm{~mm}$. By applying these recognition criteria, all the seeds were included for every image, preventing the recognition of undesirable objects in the digital image.

\subsubsection{Colour}

RGB colour was obtained from raw images, which involves minimally processed data from the CCD device. Then, CIELAB colour coordinates were obtained from the RGB values, taking into account the camera calibration. The colorimetric data of digital images (Table 1 ) entails high standard deviation values due to the chromatic heterogeneity of the samples.

The phenomenon of browning appears as a decrease of the quantitative colorimetric parameters $\left(L^{*}\right.$ and $\left.C^{*}{ }_{\mathrm{ab}}\right)$. This fact is evidenced in a darkening and a lack of chromaticity of the final samples of grape seeds. Browning advances while chemical composition changes, so it suggests a connection between these two phenomena.

Not only the quantitative psychophysical colorimetric parameters $\left(L^{*}\right.$ and $C^{*}$ ab $)$ correlated with some phenolic compounds, but also the hue $\left(h_{\mathrm{ab}}\right)$, demonstrating that the browning phenomenon implies changes also from a qualitative point of view.

Additionally, chromatic heterogeneity was measured, considering being this variable the fraction of pixels that deviate more than $10 \%$ from the average intensity. Chromatic heterogeneity increases in the early stages of maturation and decreases in the final stages, because browning process does not occur in a homogeneous manner. The seeds become dark brown bit by bit while the light green areas disappear in the same way.

\subsubsection{Morphological variables}

The spatial unit in imaging is the pixel, so for calculating the morphological data from the images we need to perform the spatial calibration to find the correspondence between pixel and millimetre. With this purpose, the image of a calliper was taken in the same way than the seeds images.

The size of the seeds was measured in two directions: diameter along major axis of the seed (length) and diameter along the axis perpendicular to the major axis (width). In order to measure the elongation degree of each seed, the aspect ratio was calculated which is the ratio between major and minor axis of the ellipse equivalent to the seed. Also the roundness value was calculated by using the following dimensionless equation:

Roundness : $\frac{\text { perimeter }^{2}}{4 \times \pi \times \text { area }}$

in such a way that a perfect circle has a roundness value of one and it becomes higher while the shape elongates.

The area of each pixel was calculated by the spatial calibration. Thus, the area of each seed was calculated by pixel count.

\subsection{Statistical treatment}

Once the chemical and image analysis were applied for all the samples, direct univariate relationships (linear regression models)

Table 1

CIELAB values of the grape seeds at each stage of maturation. Mean \pm Std. Dev. (CIELAB units.)

\begin{tabular}{|c|c|c|c|c|c|c|}
\hline & \multicolumn{6}{|l|}{ Stage } \\
\hline & I & II & III & IV & V & VI \\
\hline$L^{*}$ & $45.1 \pm 6.7$ & $43.4 \pm 6.8$ & $41.5 \pm 7.0$ & $40.3 \pm 6.9$ & $42.3 \pm 6.6$ & $40 \pm 7.3$ \\
\hline$a^{*}$ & $7.6 \pm 3.1$ & $7.6 \pm 2.9$ & $7.6 \pm 2.9$ & $8.2 \pm 3.0$ & $6.4 \pm 2.7$ & $8.4 \pm 3.0$ \\
\hline$b^{*}$ & $23.8 \pm 6.8$ & $22.1 \pm 6.5$ & $21.9 \pm 6.4$ & $21.9 \pm 6.6$ & $21.5 \pm 6.3$ & $21.4 \pm 6.7$ \\
\hline$C_{\mathrm{ab}}^{*}$ & $25.2 \pm 6.8$ & $23.6 \pm 6.4$ & $23.4 \pm 6.3$ & $23.6 \pm 6.6$ & $22.6 \pm 6.2$ & $23.2 \pm 6.7$ \\
\hline$h_{\mathrm{ab}}$ & $71.9 \pm 7.6$ & $70.3 \pm 8.2$ & $70.2 \pm 9.3$ & $68.8 \pm 7.7$ & $72.5 \pm 8.5$ & $68.0 \pm 11.2$ \\
\hline
\end{tabular}


Table 2

Correlation matrix $\left(R^{2}\right)$ between chemical composition and parameters obtained by image analysis in each stage. Results in bold are significant at $\alpha=0.05$.

\begin{tabular}{|c|c|c|c|c|c|}
\hline & Hydroxibenzoic acids & Monomers & Dimers & Trimers & Galloylated compounds \\
\hline$L^{*}$ & 0.54 & 0.93 & 0.39 & 0.35 & 0.80 \\
\hline$a^{*}$ & -0.38 & -0.18 & 0.16 & 0.41 & 0.00 \\
\hline$b^{*}$ & 0.27 & 0.91 & 0.38 & 0.46 & 0.85 \\
\hline$C^{*}{ }_{a b}$ & 0.17 & 0.83 & 0.41 & 0.55 & 0.82 \\
\hline$h_{\mathrm{ab}}$ & 0.45 & 0.57 & 0.01 & -0.18 & 0.38 \\
\hline Area & -0.52 & -0.32 & -0.88 & -0.64 & -0.55 \\
\hline Aspect & -0.42 & -0.20 & 0.03 & -0.26 & -0.03 \\
\hline Roundness & 0.00 & -0.20 & 0.31 & 0.08 & 0.01 \\
\hline Length & -0.91 & -0.59 & -0.77 & -0.81 & -0.61 \\
\hline Width & -0.10 & -0.13 & -0.57 & -0.23 & -0.36 \\
\hline Heterogeneity & 0.72 & 0.42 & 0.77 & 0.94 & 0.53 \\
\hline
\end{tabular}

Table 3

Results of forward stepwise Multiple Regression. Results in bold are significant at $\alpha=0.05$.

\begin{tabular}{|c|c|c|c|c|c|}
\hline & Beta & Std. Err. of Beta & $B$ & Std. Err. of B & $p$-level \\
\hline Intercept & & & 114.399 & 18.817 & 0.0089 \\
\hline$L^{*}$ & -0.816 & 0.196 & -0.680 & 0.164 & 0.0254 \\
\hline Length & 0.513 & 0.096 & 9.840 & 1.839 & 0.0128 \\
\hline Aspect & -0.316 & 0.131 & -11.836 & 4.918 & 0.0953 \\
\hline Roundness & -0.216 & 0.127 & -15.160 & 8.901 & 0.1871 \\
\hline$C_{\mathrm{ab}}^{*}$ & -0.182 & 0.156 & -0.329 & 0.281 & 0.3271 \\
\hline
\end{tabular}

between the chemical information and the parameters obtained by image analysis were studied. As it can be observed in Table 2, high correlations have been found between colour and phenolic composition, such as monomers and lightness $\left(L^{*}\right)\left(R^{2}=0.93\right.$, $p=0.008)$ or galloylated compounds and chroma $\left(C_{\mathrm{ab}}^{*}\right)\left(R^{2}=0.82\right.$, $p=0.047)$. Moreover, some high correlations were also found with morphological and appearance data, such as trimers and heterogeneity $\left(R^{2}=0.94, p=0.005\right)$ or hydroxybenzoic acids and length $\left(R^{2}=-0.91, p=0.011\right)$. These considerable correlations with some morphological parameters were the reason why they were also included in further multivariate analysis.

Thus, a forward stepwise multiple regression was applied for estimating the maturity stage of grape seeds from the values obtained by image analysis. The multiple regression models included sequentially lightness $\left(L^{*}\right)$, chroma $\left(C^{*}\right.$ ab $)$, seed length, roundness and aspect ratio as independent variables in the prediction, being lightness $\left(L^{*}\right)$ and length the variables which influenced significantly $(p<0.05)$ (Table 3$)$. Fig. 2 shows the scatterplot of observed vs. predicted values of maturity stage, showing linear fit with quite good correlation coefficient $\left(R^{2}=0.97, p=0.0089\right)$. This fact, together to the narrow regression bands (the confidence level of the bands represents the probability that additional sample

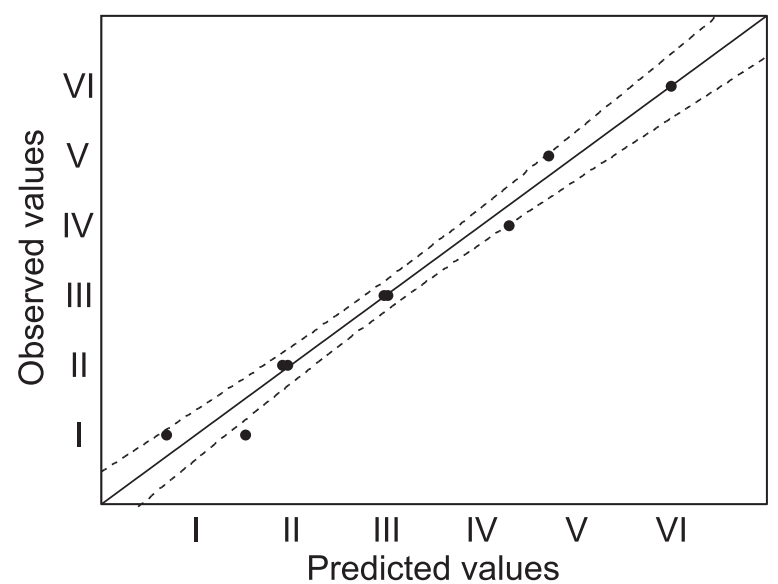

Fig. 2. Scatterplot of observed vs. predicted values of stage of maturity. Dashed lines shows confidence bands at (95\%). points will fall within the prediction interval) indicate the high predictability of the maturity stage based on image analysis data.

\section{Conclusions}

Twenty one identified phenolic compounds decreased their concentration along the different grape seeds maturation stages studied, related to polymerization reactions occurring along this period, and the colorimetric and morphological parameters analysed by image analysis also changed.

In some cases, single linear regression models show that there are good relationships between chemical and appearance data. Moreover, it is possible to estimate the maturity stage of grape seeds by applying a forward stepwise multiple regression models to these data, being achieved a correlation coefficient of $R^{2}=0.97$ for predicting the maturity stage.

The results suggest that image analysis has good potential to be used for colour and appearance measurements in grape seeds. It is not yet a substitute for conventional chemical analysis but it is an attractive alternative due to its simplicity, versatility, and low-cost.

Although the suggested method should be applied to other grape varieties to study its robustness, it is a quick and objective way to estimate the maturity stage. Controlling the variable affecting, it could become a way to assess the chemical characteristics of grape seeds during maturation, saving time and chemical reagents and thus allowing the winery to take quick decisions, for example in determining the moment of harvesting.

\section{Acknowledgements}

We thank the Ministry of Science and Innovation of Spain the financial support: Projects AGL2008-05569-C02-01 and AGL200805569-C02-02, and fellowship BES-2009-025429.

\section{References}

[1] T. Escribano-Bailón, M. Álvarez-García, J.C. Rivas-Gonzalo, F.J. Heredia, C. Santos-Buelga, J. Agric. Food Chem. 49 (2001) 1213-1217.

[2] R. Gawel, J. Aust, Grape Wine Res. 4 (1998) 74-95.

[3] E.Q. Xia, G.F. Deng, Y.J. Guo, H.B. Li, Int. J. Mol. Sci. 11 (2010) 622-646.

[4] R. Ferrer-Gallego, M. García-Marino, J.M. Hernández-Hierro, J.C. Rivas-Gonzalo, M.T. Escribano-Bailón, Anal. Chim. Acta 660 (2010) 22-28.

[5] R. Ristic, P.G. Iland, Aust. J. Grape Wine Res. 11 (2005) 43-58. 
[6] V.A.P. De Freitas, Y. Glories, A. Monique, Am. J. Enol. Vitic. 51 (2000) 397-403.

[7] J.A. Kennedy, Y. Hayasaka, S. Vidal, E.J. Waters, G.P. Jones, J. Agric. Food Chem. 49 (2001) 5348-5355.

[8] K.L. Yam, S.E. Papadakis, J. Food Eng. 61 (2004) 137-142.

[9] Commision Internationale de l'Eclairage (CIE), Technical Report, Colorimetry, CIE Central Bureau, Vienna 2004.

[10] D.G. Savakar, S.B. Anami, Int. J. Food Eng. 5 (2009) (Art. 14).

[11] C. Zheng, D.W. Sun, in: D.W. Sun (Ed.), Computer Vision Technology for Food Quality Evaluation, Academic Press, Amsterdam, 2008, pp. 57-80.

[12] H.H. Wang, D.W. Sun, J. Food Sci. 67 (2002) 745-749.

[13] C. Zheng, D.W. Sun, in: D.W. Sun (Ed.), Computer Vision Technology for Food Quality Evaluation, Academic Press, Amsterdam, 2008, pp. 37-56.

[14] K. Jack, Digital Video, D.S.P. Newnes, Burlington (2008) 15-29.

[15] M. García-Marino, J.C. Rivas-Gonzalo, E. Ibáñez, C. García-Moreno, Anal. Chim. Acta 563 (2006) 44-50.
[16] S. de Pascual-Teresa, J.C. Rivas-Gonzalo, C. Santos-Buelga, Int. J. Food Sci. Technol. 35 (2000) 33-40.

[17] M.T. Escribano-Bailón, Y. Gutiérrez-Fernández, J.C. Rivas-Gonzalo, C. SantosBuelga, J. Agric. Food Chem. 40 (1992) 1794-1799.

[18] M.R. Luo, F.H. Cui, C. Li, British Patent Entitled Apparatus and Method for Measuring Colour (DigiEye System), Derby University Enterprises Limited, 2001 (0124683.4).

[19] F.J. Heredia, M.L. Gonzalez-Miret, C. Álvarez, A. Ramírez, DigiFood [No. SE01298], 2006.

[20] J.A. Kennedy, M.A. Matthews, A.L. Waterhouse, Phytochemistry 55 (2000) 77-85.

[21] M.O. Downey, J.S. Harvey, S.P. Robinson, Aust.J. Grape Wine Res. 9(2003) 15-27.

[22] A.M. Jordao, J.M. Ricardo Da Silva, O. Laureano, Am. J. Enol. Vitic. 52 (2001) 230-234.

[23] V. Núñez, C. Gómez-Cordovés, B. Bartolomé, Y.J. Hong, A.E. Mitchell, J. Sci. Food Agric. 86 (2006) 915-921. 